Article

\title{
Combined Conformal Strongly-Coupled Magnetic Resonance for Efficient Wireless Power Transfer
}

\author{
Matjaz Rozman ${ }^{1}$, Michael Fernando ${ }^{1}$, Bamidele Adebisi ${ }^{1, *}$, Khaled M. Rabie ${ }^{1}$, Rupak Kharel ${ }^{1}$, \\ Augustine Ikpehai ${ }^{1}$ and Haris Gacanin ${ }^{2}$ \\ 1 School of Engineering, Manchester Metropolitan University, Manchester M1 5GD, UK; \\ matjaz.rozman@stu.mmu.ac.uk (M.R.); m.fernando@mmu.ac.uk (M.F.); k.rabie@mmu.ac.uk (K.M.R.); \\ r.kharel@mmu.ac.uk (R.K.); augustine.ikpehai@stu.mmu.ac.uk (A.I.) \\ 2 Nokia Bell Labs Copernicuslaan 50, 2018 Antwerp, Belgium; haris.gacanin@nokia-bell-labs.com \\ * Correspondence: b.adebisi@mmu.ac.uk; Tel.: +44-7985-399-832
}

Academic Editor: Hongjian Sun

Received: 7 December 2016; Accepted: 4 April 2017; Published: 7 April 2017

\begin{abstract}
This paper proposes a hybrid circuit between a conformal strongly-coupled magnetic resonance (CSCMR) and a strongly-coupled magnetic resonance (SCMR), for better wireless power transmission (WPT). This combination promises to enhance the flexibility of the proposed four-loop WPT system. The maximum efficiency at various distances is achieved by combining coupling-matching between the source and transmitting coils along with the coupling factor between the transmitting and receiving coils. Furthermore, the distance between transmitting and receiving coils is investigated along with the distance relationship between the source loop and transmission coil, in order to achieve the maximum efficiency of the proposed hybrid WPT system. The results indicate that the proposed approach can be effectively employed at distances comparatively smaller than the maximum distance without frequency matching. The achievable efficiency can be as high as $84 \%$ for the whole working range of the transmitter. In addition, the proposed hybrid system allows more spatial freedom compared to existing chargers.
\end{abstract}

Keywords: conformal strongly-coupled magnetic resonance (CSCMR); strongly-coupled magnetic resonance (SCMR); efficiency; flexibility; matching; wireless power transfer (WPT)

\section{Introduction}

Wireless power transfer (WPT) technology is capable of charging electronic devices wirelessly eliminating the traditional cable requirement. This technology is not only more convenient, but can also enhance the health and safety within both domestic and commercial environments. Although WPT can be realized using either inductive coupling or radio frequency (RF) signals (see e.g., [1-3]), the former will be the focus of this paper.

Recently, a great number of attempts have been made towards the deployment of inductive WPT systems, since low energy density batteries are no longer sufficient to saturate the escalating energy demand of the electronic apparatus [3,4]. The need for an industry standard for the interoperability of the wireless devices was elevated simultaneously with the increasing number of wireless charging efforts. As a result, the Qi standard was introduced by the wireless power consortium and was adopted for both low and medium wireless power charging specifications during the last few years [5-7]. This standard is based on a tightly coupled two-coil system supporting charging up to 5 W. However, the efficiency of the two-coil system fluctuates upon the coil alignment and the distance between the transmitting coil (Tx) and receiving coil ( $\mathrm{Rx})[8,9]$. The two-coil charging system has also been proposed for numerous appliances, especially for electric vehicle (EV) charging applications [10,11]. 
In order to maximize the distance between the Tx and $R x$, the strongly-coupled magnetic resonance (SCMR) method was introduced in 2007 [12]. The Authors of this study pointed out that this method is capable of wirelessly transferring $60 \mathrm{~W}$ of electrical power over a distance of $2 \mathrm{~m}$ with $40 \%$ efficiency. Moreover, the SCMR system has demonstrated higher efficiencies by designing the transmitting and receiving resonant loops to resonate at the same frequency, where they exhibit the maximum quality factor [13]. However, the fact that the SCMR system has a high sensitivity to the misalignment between the coils [14,15] and its bulky structure are two main drawbacks of this system. In order to overcome these drawbacks, the conformal SCMR (CSCMR) concept was introduced in [16]. In this method, both the transmitter and receiver are embedded in the same plane with Tx and Rx resonators. A few novel designs of the CSCMR system are also built on the printable version; see, e.g., [17-19].

Critically coupled resonators are required in order to achieve maximum efficiency of SCMR systems [20,21]. Thus, the resonant frequency of the Tx and Rx loop varies due to the air gap between resonators. The impedance matching technique was implemented in [22,23], while [24] recites the adaptive matching method implemented to maintain the maximum level of system efficiency. Furthermore, in [25], a transmission coil array technique was proposed where appropriate phase weights of the transmission circuit are used according to the energy deposition profile of the receiving coil. However, in previous research studies, it is assumed that the coupling coefficient between source and Tx loop is fixed; therefore, the system performance was analysed upon multiple distances between Tx and Rx loops.

Misalignment and angle alignment also play a crucial role in the efficiency of WPT systems. For instance, the authors in [26-28] explored the impact of the misalignment and angle alignment on the two coils, which are magnetically coupled in a resonant wireless power system. In addition, this study proposed algorithms capable of adjusting the transmitting frequency to achieve maximum efficiency. Moreover combining the two systems will simultaneously reduce the bulkiness of the receiver coils while enhancing the spatial freedom at the transmitting end.

In this paper, we explore the possibility of maintaining the maximum efficiency of four loop inductive coupling method at any distance between the Tx and Rx loops by either reducing or increasing the gap between the source and Tx loops. To the best of our knowledge, no existing work has considered this in the open literature. This will allow more spatial freedom in terms of receiver positioning without using frequency matching algorithms, since the system frequency remains constant. This may also reduce the cost of the power source, since it can be designed to operate at a single frequency. Therefore, the contributions of this paper are as follows. We first derive an analytical model of combined SCMR-CSCMR system, which simultaneously enhances the flexibility and usability of both models. Additionally, the relationship between the source and Tx loop gap is studied, in relation to the gap between the Tx and Rx resonating loops along with the effect of the loop dimensions. The second contribution of this work resides in conducting an experiment to validate the analytical results. Furthermore, a novel concept for flexible charging will be introduced where the receiver position can be adjusted to enhance the maximum efficiency without using multiple coils or adjusting the frequency. Results demonstrate that the proposed system is able to attain higher efficiencies compared to frequency matching, being further close to the maximum. This further indicates that the system performance was least affected by the combined SCMR-CSCMR method or by the adjustments to the distance between the source loop and Tx.

The rest of the paper is structured as follows. Section 2 describes the proposed hybrid WPT system model. In Section 3, a mathematical analysis is presented providing an insight into the subject using multiple theoretical equations, while the system specifications are presented in Section 4. In Section 5, the experimental parameters and the system configuration are further described. The comparison between calculated and experimental setup is discussed in Section 5, and the system efficiency upon the angle and misalignment of the system is presented in Section 6. Finally, the conclusions are drawn in Section 7. 


\section{System Model of the Proposed Hybrid WPT System}

The hybrid WPT model proposed in this study is shown in Figure 1. This system merges two different wireless transmission system concepts. The transmitting end is designed using the SCMR concept, where the source loop is placed outside the Tx loop in order to provide the flexibility of changing distance between the two loops. On the other hand, the receiver implementation is based on the CSCMR concept, where the load loop is placed concentrically inside the Rx loop. This concept significantly shrinks the size of the receiver end while the coupling between the two coils remains fixed.

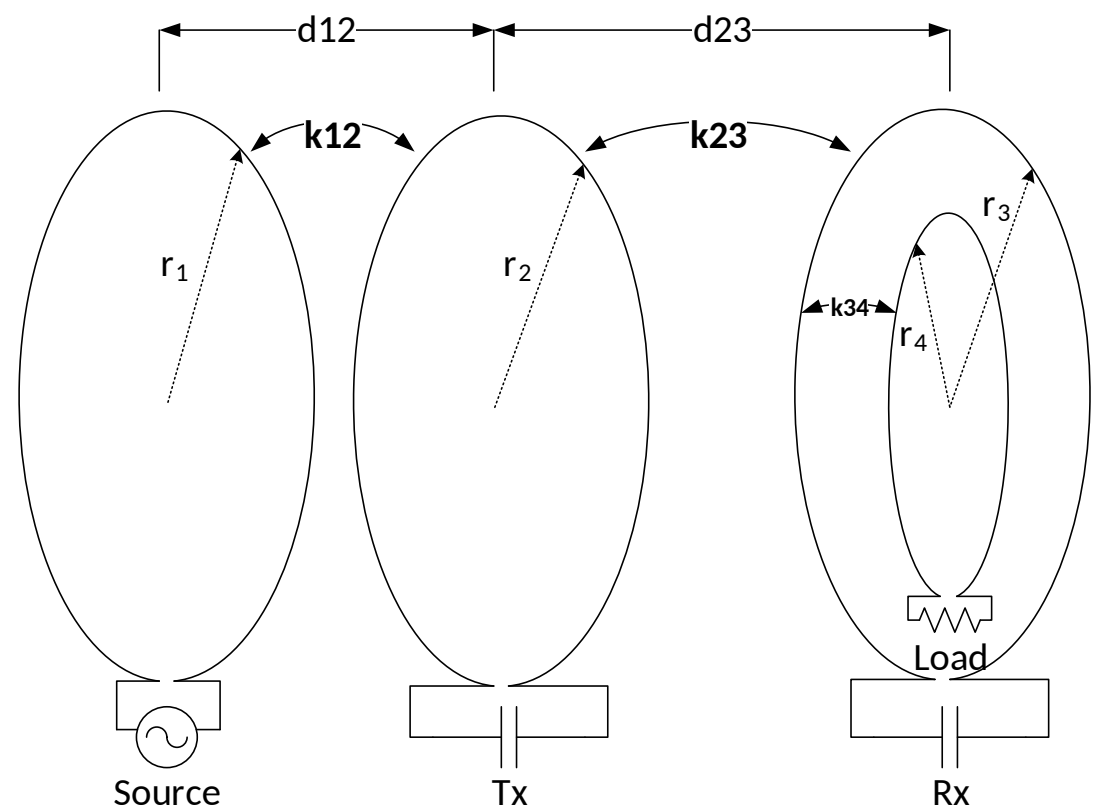

Figure 1. Combined SCMR-CSCMR system.

For instance, when the distance between Tx and $\mathrm{Rx}\left(d_{23}\right)$ is changed, the coupling factor $\left(k_{23}\right)$ varies accordingly. In order to compensate the corresponding change to maximize the system efficiency, the coupling factor $\left(k_{12}\right)$ must be simultaneously altered. According to the CSCMR concept, the coupling factor $\left(k_{34}\right)$ remains fixed; hence, only the coupling factor $k_{12}$ can be changed by adjusting the distance between the source and Tx loops $\left(d_{12}\right)$. Therefore, a relationship between the two distances $\left(\frac{\triangle d_{23}}{\triangle d_{12}}\right)$ has to be expressed.

\section{Theoretical Analysis of Strongly-Coupled WPT Systems}

Both conventional CSCMR and SCMR approaches consist of four loops, namely source loop, load loop, Tx and Rx loops. Both Tx and Rx loops are designed to oscillate at the same frequency, and therefore, the quality factor $(\mathrm{Q})$ between the two loops is maximized. This ensures that the power transmission between these loops is maximal while the loops are connected together via electromagnetic coupling. The system can be described by an equivalent circuit diagram as shown in Figure 2, which illustrates the hybrid systems with two pairs of resonators. From it, a four-port network model can be derived, based on Kirchoff's voltage law $[29,30]$. 


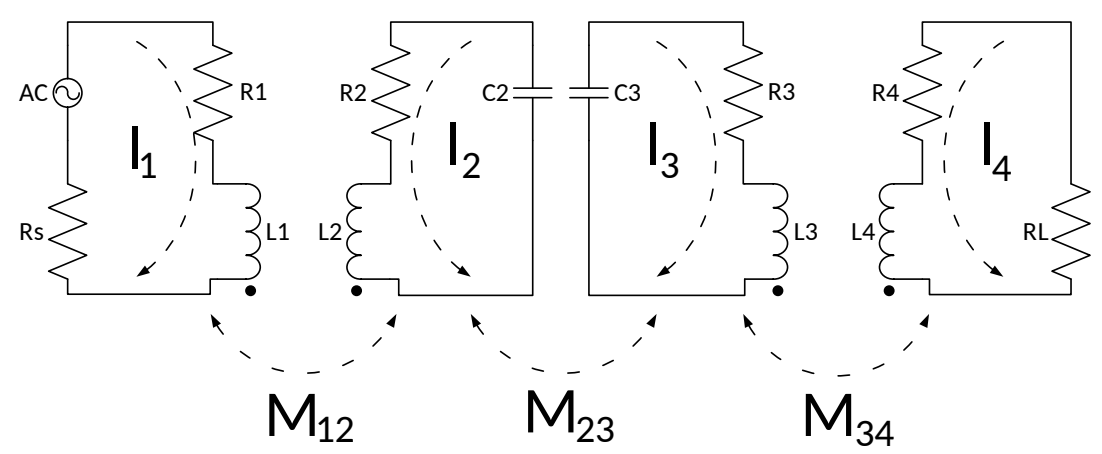

Figure 2. Equivalent hybrid SCMR and CSCMR circuit diagrams.

The electromagnetic induction field $(\mathrm{H})$ for one of the parallel coaxial loops can, as follows based on Ampere's law and Faraday's electromagnetic induction law [31,32], be shown as:

$$
H_{y}=\int \Delta H_{y}=\frac{I_{x} r_{y}^{2}}{2\left(r_{y}^{2}+d^{2}\right)^{\frac{3}{2}}}
$$

where $d$ represents the distance between the two loops, $r_{y}$ represents the radius of $y$-th loop and $I_{x}$ is the current through the $x$-th loop. It is worth noting that Equation (1) is more accurate at short distances. However, in order to simplify the analysis in this study, we approximate the mutual inductance (M) between the $x$-th and $y$-th loops including the area enclosed by the coil [33,34], as:

$$
M_{x y}=\frac{\mu_{0} H_{y} A_{y}}{I_{x}}=\frac{\mu_{0} r_{x}^{2} r_{y}^{2} \pi}{2 \sqrt{\left(r_{y}^{2}+d^{2}\right)^{3}}}
$$

where $r_{x}$ and $r_{y}$ represent the radius of the $x$-th and $y$-th loops, respectively and $\mu_{0}$ donates the permeability of free space. $A_{y}$ stands in for the area of the $y$-th loop and can be mathematically expressed as $A_{y}=\pi r_{y}^{2}$.

For the Rx elements of the CSCMR system, which consist of two coaxial loops placed concentrically on the same plane, the magnetic field is usually attributed to the larger loop. Therefore, when the distance $d_{34}=0$, the mutual inductance can be simplified as:

$$
M_{x y}=\frac{\mu_{0} r_{x}^{2} \pi}{2 r_{y}}
$$

where $r_{x} \ll r_{y}$. A coupling coefficient between the two loops can be expressed as:

$$
k_{x y}=\frac{M_{x y}}{\sqrt{L_{x} L_{Y}}} .
$$

The current in each circuit can be calculated by applying Kirchoff's voltage law. Therefore, the scattering parameter $S_{21}$, which represents the ratio between the signal at the output port and the injected signal at the input, can be calculated as:

$$
S_{21}=2 \frac{V_{L}}{V_{S}}\left(\frac{R_{S}}{R_{L}}\right)^{\frac{1}{2}}
$$

Combined with Equation (4), the $S_{21}$ parameter can be given as:

$$
S_{21}=\frac{A}{B+C+D+E+F}
$$


where:

$$
\left\{\begin{array}{l}
A=j 2 \omega^{3} k_{12} k_{23} k_{34} L_{2} L_{3} \sqrt{L_{1} L_{4} R_{S} R_{L}} \\
B=Z_{1} Z_{2} Z_{3} Z_{4} \\
C=k_{12}^{2} L_{1} L_{2} Z_{3} Z_{4} \omega^{2} \\
D=k_{23}^{2} L_{2} L_{3} Z_{1} Z_{4} \omega^{2} \\
E=k_{34}^{2} L_{3} L_{4} Z_{1} Z_{2} \omega^{2} \\
F=k_{12}^{2} k_{34}^{2} L_{1} L_{2} L_{3} L_{4} \omega^{4}
\end{array}\right.
$$

and $Z_{1}, Z_{2}, Z_{3}$ and $Z_{4}$ are the loop impedances of the four coils and can be calculated as:

$$
Z_{x}=R_{x}+j \omega L_{x}-j \frac{1}{\omega C_{x}}
$$

where $R_{x}, L_{x}$ and $C_{x}$ represent total resistance, the equivalent inductance and external capacitance of the $x$-th loop, respectively.

In order for the system to function at its maximum efficiency, the resonant frequency can be calculated as:

$$
\eta=\frac{P_{\text {LOAD }}}{P_{\text {SOURCE }}}=\frac{\left|S_{21}\right|^{2}}{\left(1-\left|S_{11}\right|^{2}\right)}
$$

For the system to work at maximum efficiency, resonant frequency $\left(f_{0}\right)$ is calculated as:

$$
f_{0}=\frac{1}{2 \pi \sqrt{(L C)}}
$$

It is also important that the $Q$ of the Tx and the Rx loops be high [30]. The $Q$ of the $y$-th loop can be expressed as:

$$
Q_{y}=\frac{\omega_{0} L_{y}}{R_{y}}
$$

where $\omega_{0}$ denotes the angular frequency, which can be calculated as $\omega_{0}=\omega_{y}=\frac{1}{\sqrt{L_{y} C_{y}}}$ in terms of $\left[\frac{\mathrm{rad}}{\mathrm{s}}\right]$.

\section{Numerical Analysis}

The efficiency of both the CSCMR and SCMR systems strongly depend on the geometrical parameters of the loops. The above system has been designed to have its maximum operating distance of $12 \mathrm{~mm}$ when it operates at a frequency of 7.2 MHz. The radius of the source loop, Tx loop and Rx loop, $\left(r_{1}=r_{2}=r_{3}\right)$, was calculated from Equations (2) and (3) and set at $30 \mathrm{~mm}$. As calculated in [35], the system will achieve the maximum distance when the coupling factor between $\mathrm{Rx}$ and load loops $\left(k_{34}\right)$ is equal to 0.1 . To ensure that the coupling factor $k_{34}$ matches the requirements, the radius of the load loop $\left(r_{4}\right)$ was calculated as $5 \mathrm{~mm}$, and its inductance was set as $L_{4}=0.19 \mu \mathrm{H}$. In addition, to ensure that the $\mathrm{Q}$ requirement between the two loops is fulfilled, the inductance of $\mathrm{Rx}$ is set to $L_{3}=0.739 \mu \mathrm{H}$. From Equation (9), the capacitance of the Rx loop can be calculated as $C_{3}=661.2 \mathrm{pF}$, so that the required loop resonating frequency is achieved.

The inductance of the Tx loop $\left(L_{2}\right)$ was set at $2.402 \mu \mathrm{H}$ to ensure that the coupling factor and quality factor requirements are met. The desired capacitance of the Tx loop was calculated from Equation (9) and set at $C_{2}=203.42 \mathrm{pF}$ to ensure that the requirements for resonant frequency are met. The calculated values of the loop elements are presented in Table 1. Figure 3 shows that the maximum efficiency of $84 \%$ is obtained for the system when the distance between the Tx and $\mathrm{Rx}$ is equal to $12 \mathrm{~mm}$. In this case, the distance between the source and Tx loop is equal to $3.4 \mathrm{~mm}$, and therefore, the coupling factor $k_{12}$ is equal to 0.1 . Figure 3 also demonstrates the frequency splitting phenomenon 
when the load power split from a single peak to a double peak. The phenomenon is an increasing coupling factor between the Rx and Tx loops when the distance between them $\left(d_{23}\right)$ decreases to a critical point.

Table 1. Calculated values of the loops elements.

\begin{tabular}{ccc}
\hline $\boldsymbol{R}_{S} ; \boldsymbol{R}_{L}=\mathbf{5 0}$ & $r_{\mathbf{1}}=\boldsymbol{r}_{\mathbf{2}}=\boldsymbol{r}_{\mathbf{3}}=\mathbf{3 0} \mathbf{\mathbf { m m }}$ & $\boldsymbol{r}_{\mathbf{4}}=\mathbf{5} \mathbf{~ \mathbf { m }}$ \\
\hline$R_{1}=0.015 \Omega$ & $C_{1}=525.97 \mathrm{pF}$ & $L_{1}=0.929 \mu \mathrm{H}$ \\
$R_{2}=0.03 \Omega$ & $C_{2}=203.42 \mathrm{pF}$ & $L_{2}=2.402 \mu \mathrm{H}$ \\
$R_{3}=0.02 \Omega$ & $C_{3}=661.2 \mathrm{pF}$ & $L_{3}=0.739 \mu \mathrm{H}$ \\
$R_{4}=0.012 \Omega$ & $C_{4}=2571.1 \mathrm{pF}$ & $L_{4}=0.19 \mu \mathrm{H}$ \\
\hline
\end{tabular}

Our analytical model was used to study the impact on the system efficiency due to the alternations of the distances $d_{23}$ and $d_{12}$ on the efficiency of the system. In addition, it was reviewed how the impact can be used to maintain the maximum efficiency at various distances between Tx and Rx without altering the transmitting frequency.

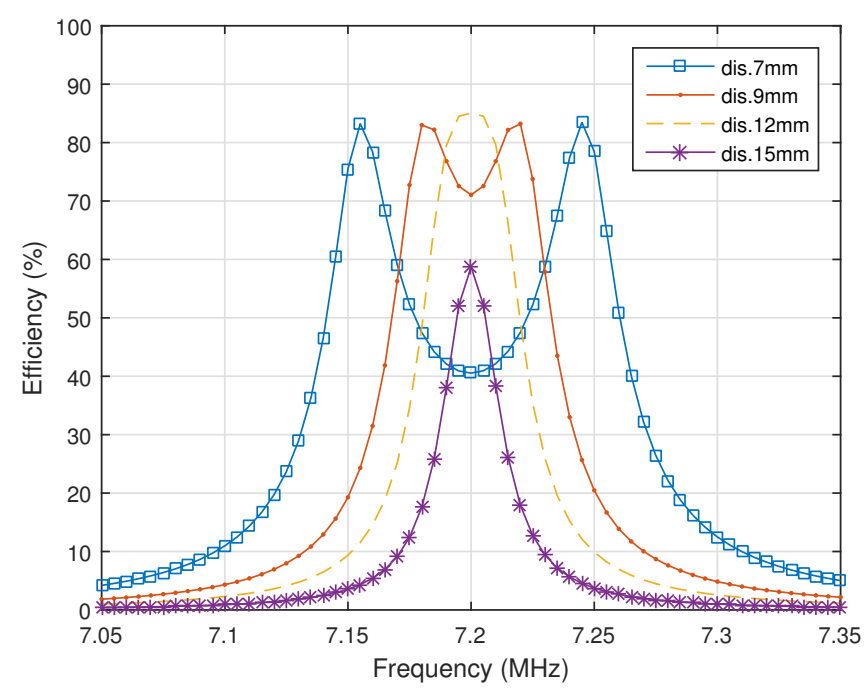

Figure 3. Maximum distance and frequency splitting phenomenon of the calculated system.

From Figure 3, it is also evident that the maximum efficiency appears when the distance between $\mathrm{Tx}$ and $\mathrm{Rx}$ loops is equal to $12 \mathrm{~mm}$, and therefore, the coupling factor between them can be calculated as $k_{23}=0.0045$, using Equations (2) and (4). The coupling factor between the source loop and Tx is equal to 0.1 . From the two known coupling factors, the relationship between distances $d_{12}$ and $d_{23}$ can be calculated from Equations (2)-(4) as:

$$
\frac{k_{12}}{k_{23}}=\frac{\left(\frac{\mu_{0} r_{1}^{2} r_{2}^{2} \pi}{2 \sqrt{\left(r_{2}^{2}+d_{12}\right)^{3}}}\right) \sqrt{L_{2} L_{3}}}{\left(\frac{\mu_{0} r_{2}^{2} r_{3}^{3} \pi}{2 \sqrt{\left(r_{3}^{2}+d_{23}\right)^{3}}}\right) \sqrt{L_{1} L_{2}}}
$$

After substituting all of the known variables into Equation (12), the relationship between the distances $d_{12}$ and $d_{23}$ can be derived as:

$$
d_{12}=\frac{0.355689 r_{2} d_{23} L_{3}^{\left(\frac{1}{6}\right)}}{r_{1}^{\left(\frac{1}{3}\right)} r_{3}^{\left(\frac{2}{3}\right)} L_{1}^{\left(\frac{1}{6}\right)}}
$$


The relationship between the distances in Equation (13) is simulated in MATLAB, and the obtained results are presented in Figure 4. It is evident that with the right calculation of the relationship between $d_{12}$ and $d_{23}$, the maximum power transmission of the SCMR system can be also achieved at comparably shorter distances than the system's maximum range. Eminently, the transmission frequency remains unaffected within the whole transmission range, which eliminates the necessity of matching circuits and tuning capacitors [36]. Figure 5 represents the maximum efficiency of the system if different load and source resistances are applied. While the resistance of the source does not have a big impact on maximum efficiency, the impact of increasing load resistance is more significant. In order to improve the performance, where the impedance of the source loop does not match the impedance of the load loop, various impedance matching techniques can be used [37,38]. However, the matching algorithms are not compulsory in this study since this experiment was carried out using a signal generator and spectrum analyser with the source and the load having $50 \Omega$ input impedances.

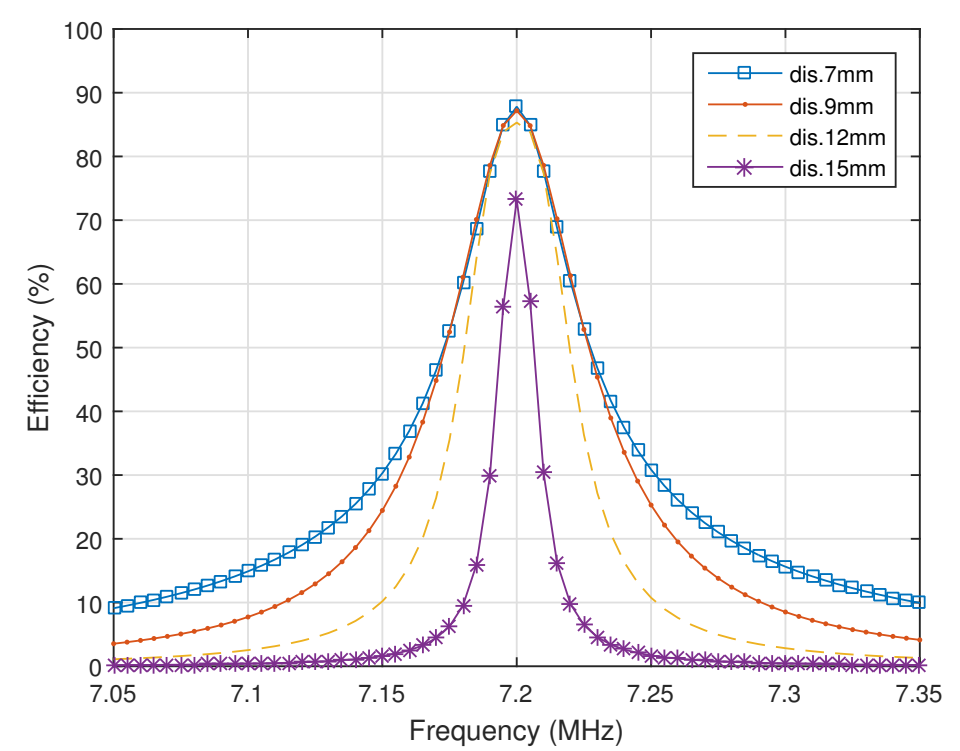

Figure 4. Simulated efficiency of the hybrid system using the calculated equation for the distance $\left(d_{12}\right)$.

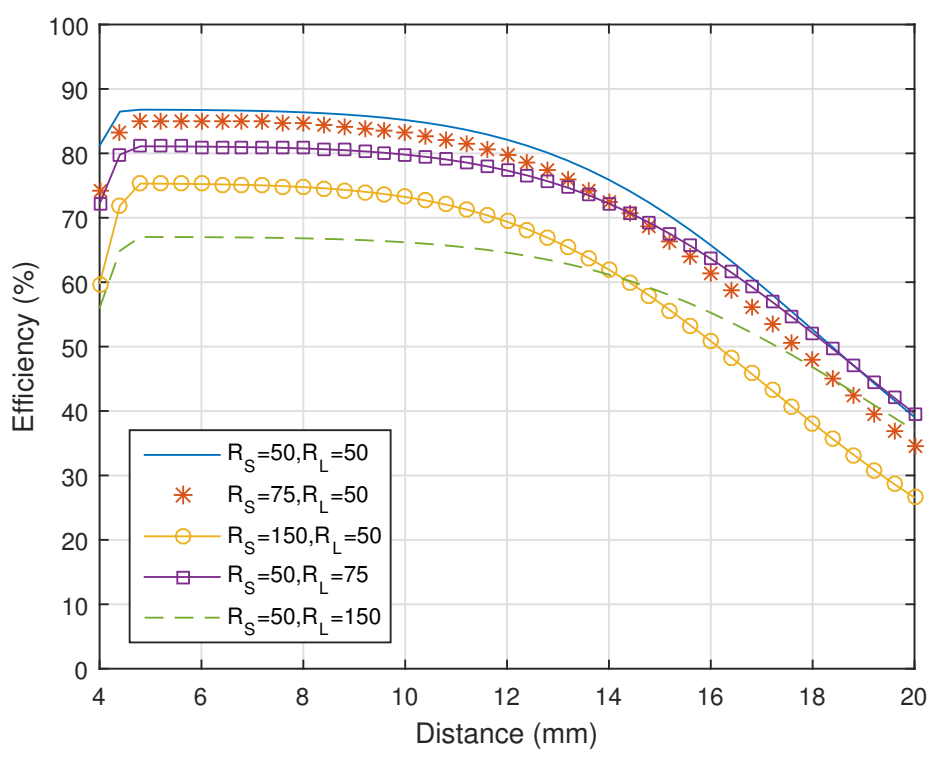

Figure 5. Maximum efficiency simulated results if the source and load resistance varies. 


\section{Experimental Setup}

In this section, an experimental setup is presented and is then utilized to explore the impact of various system parameters on the performance. In order to verify the calculated results, an appropriate system was built to comply with the calculations' model. The two loops forming the transmitter have the same size forming the SCMR system design, and the other two loops are embedded, forming the CSCMR design receiver, as shown in Figure 6.

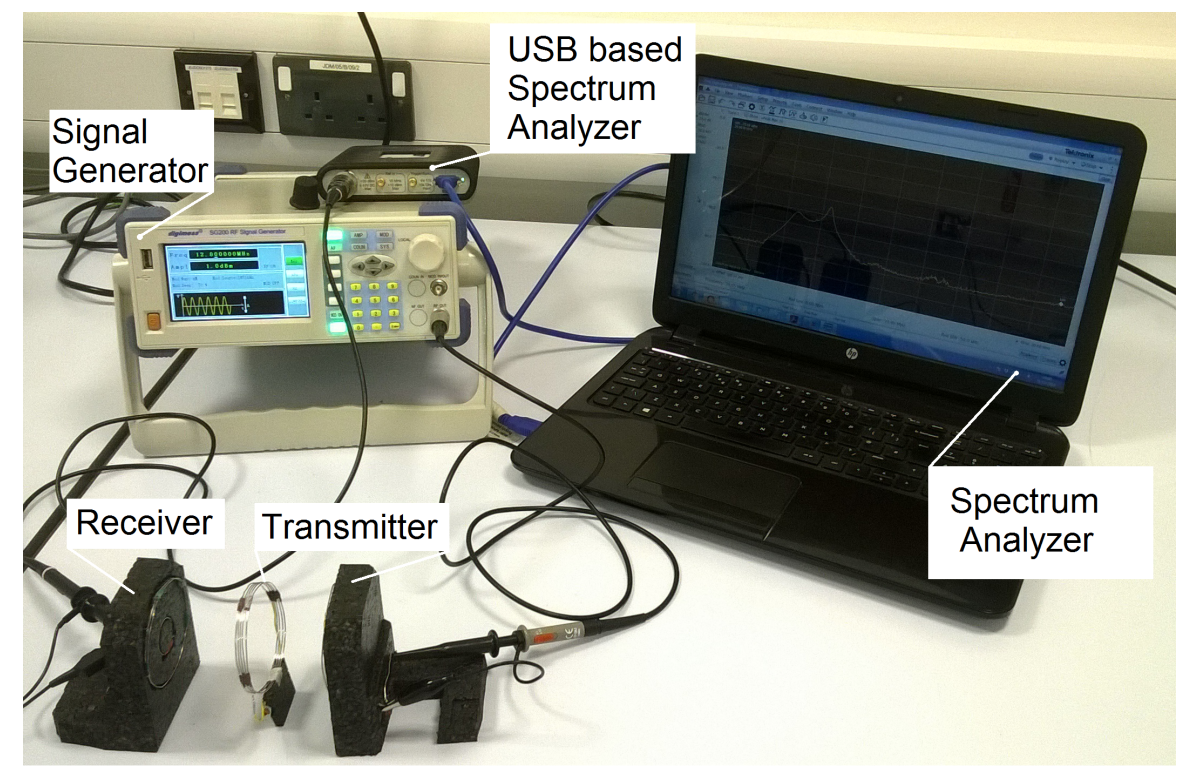

Figure 6. Practical implementation of the combined CSMR-SCSMR approach.

\subsection{System Design and Measurement}

The transmitter consists of the source and TX loops. The source loop is made of a 2.3-turn, silver-plated copper wire, while the Tx loop is made of the same wire and contains 5 turns. The receiver side of the system is made using the same wire where the Rx loop contains 1.6 turns, and the load loop is made of 1.2 turns of the wire. Ceramic capacitors were used to tune the loops to the same oscillating frequencies.

For measurement purposes, a signal generator was used to send various frequencies to the source loop, while the response was measured using a USB base spectrum analyser. Finally, the measured results were displayed using computer-based software communicating with the spectrum analyser.

\subsection{System Maximum Efficiency}

According to the calculation, an optimal design appears when the distance $d_{12}$ is equal to $3.4 \mathrm{~mm}$. In this scenario, the maximum efficiency can be achieved when the distance $d_{23}$ is equal to $12 \mathrm{~mm}$. The graphical representation in Figure 7 is plotted using the measurements and exhibits the maximum efficiency at a distance equal to $11.8 \mathrm{~mm}$. The maximum efficiency of the obtained experimental setup is $79 \%$, which is comparable, to some extent, to the $84 \%$ efficiency calculated in Section 2 . The difference can be attributed to the displacement, the shapes of the coils and variation in the electronic components. The instrument used during the testing process can also have effects on the experiment.

Based on the comparison between the measurements and the calculations, the designed system consists of a resonant frequency $20 \mathrm{kHz}$ higher than the calculated one and it also appears to be at $7.22 \mathrm{MHz}$, as shown in Figure 8. The difference in the oscillating frequencies between the calculated and measured systems appears due to the tolerance of the elements that are used in the practical application and mathematical formula approximation. In addition, the inductors that were used to fulfil the experimental purpose may also have different inductance values than the calculated values. 


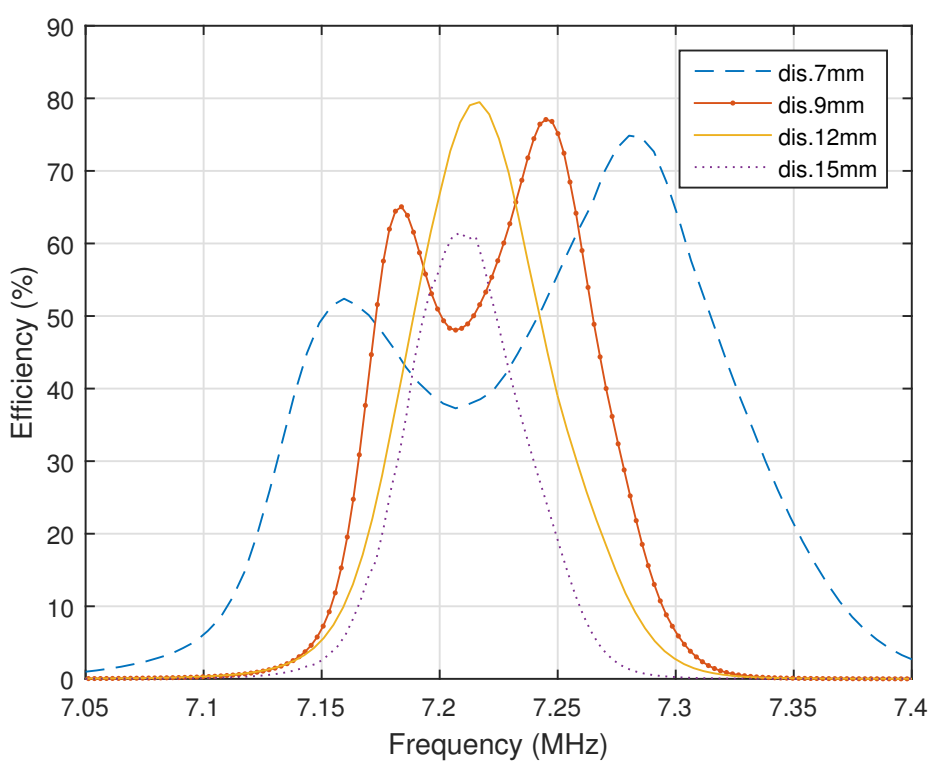

Figure 7. MATLAB converted results in transmission efficiency (\%).

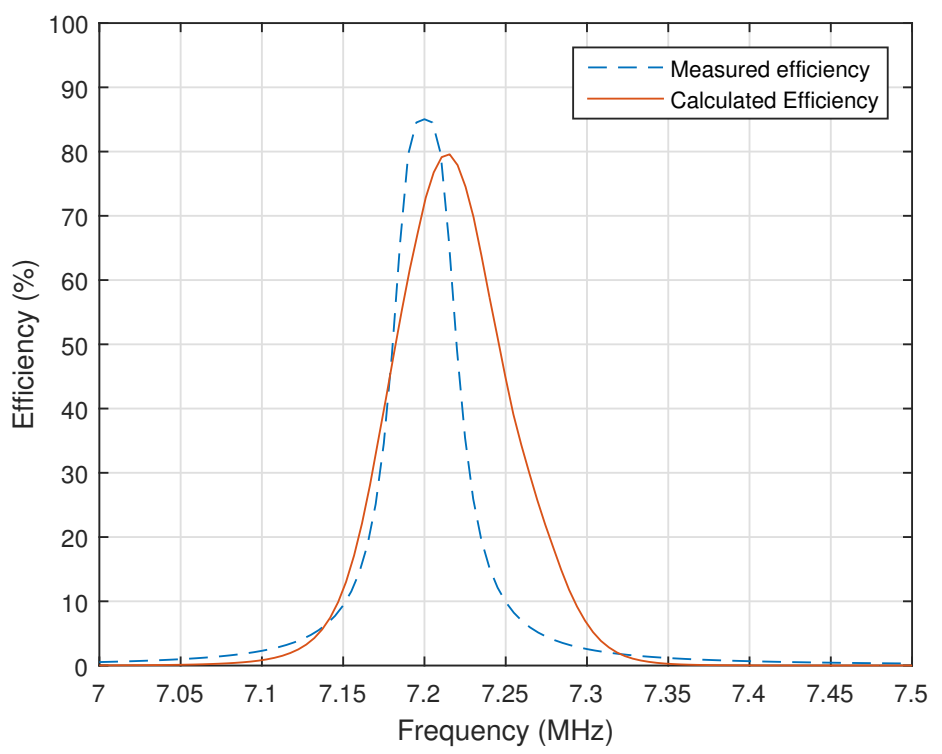

Figure 8. Frequency misalignment between the mathematical model at 7.2 $\mathrm{MHz}$ and the experimental setup at 7.22 $\mathrm{MHz}$.

\subsection{Combined System Efficiency}

In order to achieve the maximum efficiency, the distance between the source loop (S) and Tx has to be adjusted according to the distance between the Tx and Rx loops as calculated in Equation (12). As presented in Figure 9, the displacement $\Delta d_{23}$ between the Tx and $\mathrm{Rx}$ loops was compensated by adjusting the distance between $\mathrm{S}$ and $\mathrm{Tx}$ for $\Delta d_{12}$ in order to achieve the maximum efficiency. The $\mathrm{RX}$ loop in Figure 9 represents the receiver loop ( $\mathrm{Tx}$ ) with the embedded load loop, and $d_{23 \max }$ represents the maximum distance between the transmitting and receiving loops. Figure 10 illustrates a set of measurements commissioned via a combined CSMR-SCSMR system, which exhibits the maximum efficiency over higher distances. 


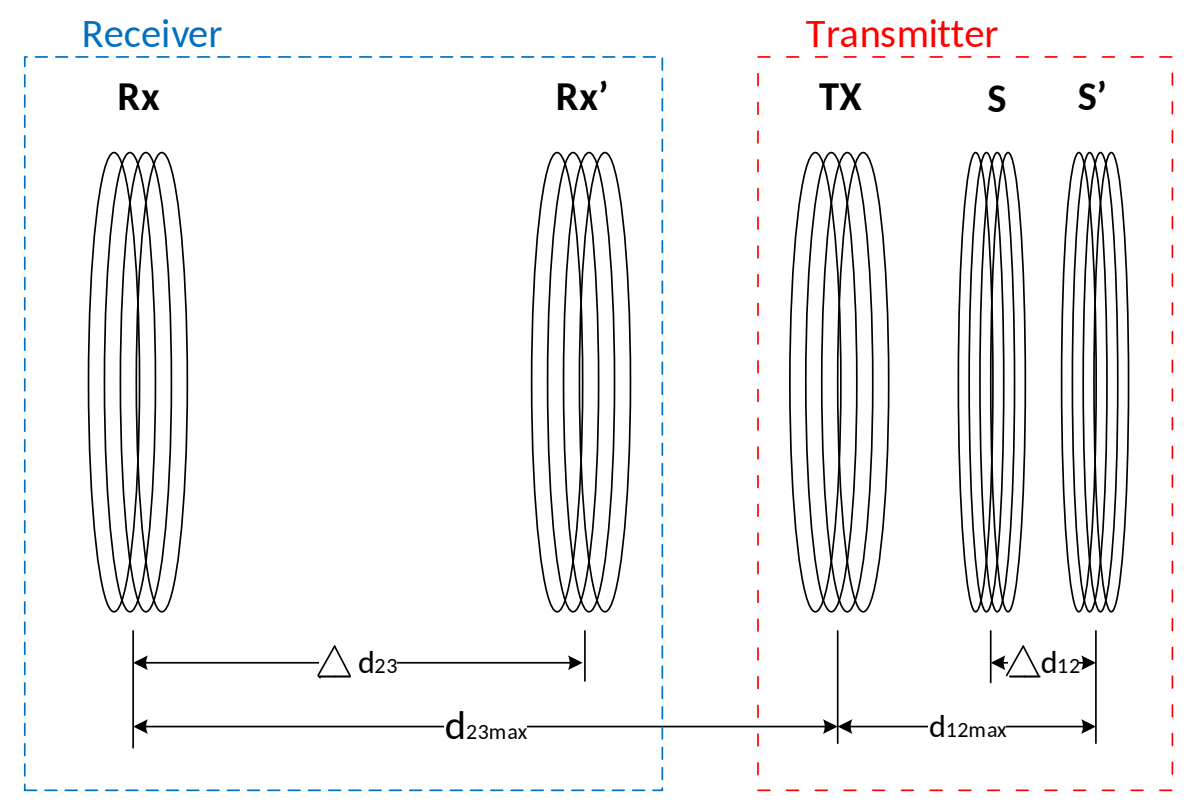

Figure 9. Maximum efficiency measurements setup.

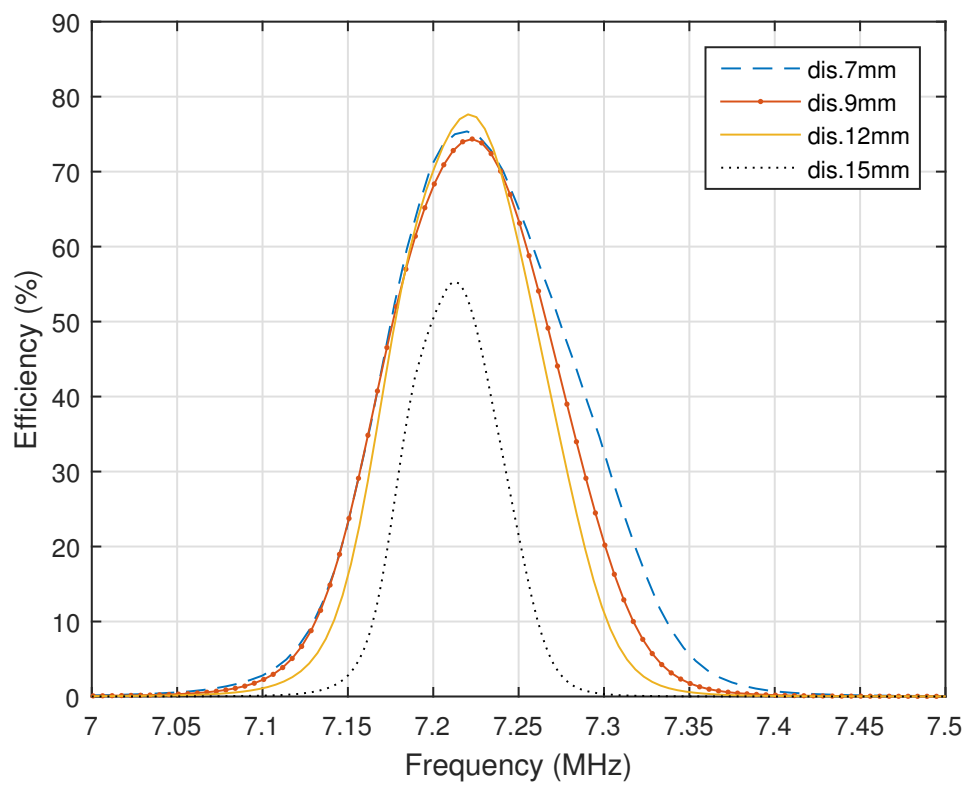

Figure 10. Measured combined SCMR-CSCMR system the holding maximum efficiency at a frequency of $7.23 \mathrm{MHz}$.

In order to justify the calculated results, the maximum distance $d_{12}$ was measured at the point where the system demonstrated its maximum efficiency along with the random distance $d_{23}$. A numerical comparison between the measured and calculated results is presented in Table 2. 
Table 2. Comparison between the measured and calculated distances $\left(d_{12}\right)$ and variation between the two in \%.

\begin{tabular}{cccc}
\hline $\boldsymbol{d}_{\mathbf{2 3}}(\mathbf{m m})$ & Calculated $\boldsymbol{d}_{\mathbf{1 2}}(\mathbf{m m})$ & Measured $\boldsymbol{d}_{\mathbf{1 2}} \mathbf{( \mathbf { m m } )}$ & Variation $\mathbf{( \% )}$ \\
\hline 3 & 1 & 1 & 0 \\
4 & 1.3 & 1 & 23 \\
5 & 1.6 & 1.5 & 6.2 \\
6 & 1.9 & 1.8 & 5.2 \\
7 & 2.2 & 2 & 10 \\
8 & 2.5 & 2.3 & 8 \\
9 & 2.8 & 2.6 & 7.1 \\
10 & 3.1 & 2.9 & 6.4 \\
11 & 3.4 & 3.2 & 5.8 \\
12 & 3.7 & 3.6 & 2.7 \\
13 & 4 & 4.1 & 0.4 \\
14 & 4.3 & 4.5 & 4.6 \\
15 & 4.6 & 4.9 & 6.5 \\
16 & 4.9 & 5 & 2 \\
17 & 5.2 & 5.3 & 1.9 \\
18 & 5.5 & 5.6 & 1.8 \\
19 & 5.8 & 5.9 & 1.7 \\
20 & 6.1 & 6.1 & 0 \\
21 & 6.4 & 6.1 & 4.6 \\
\hline
\end{tabular}

Figure 11 shows a graphical presentation of a comparison between the random distance $d_{23}$ and the measured value of $d_{12}$ against the calculated value, $d_{12}$.

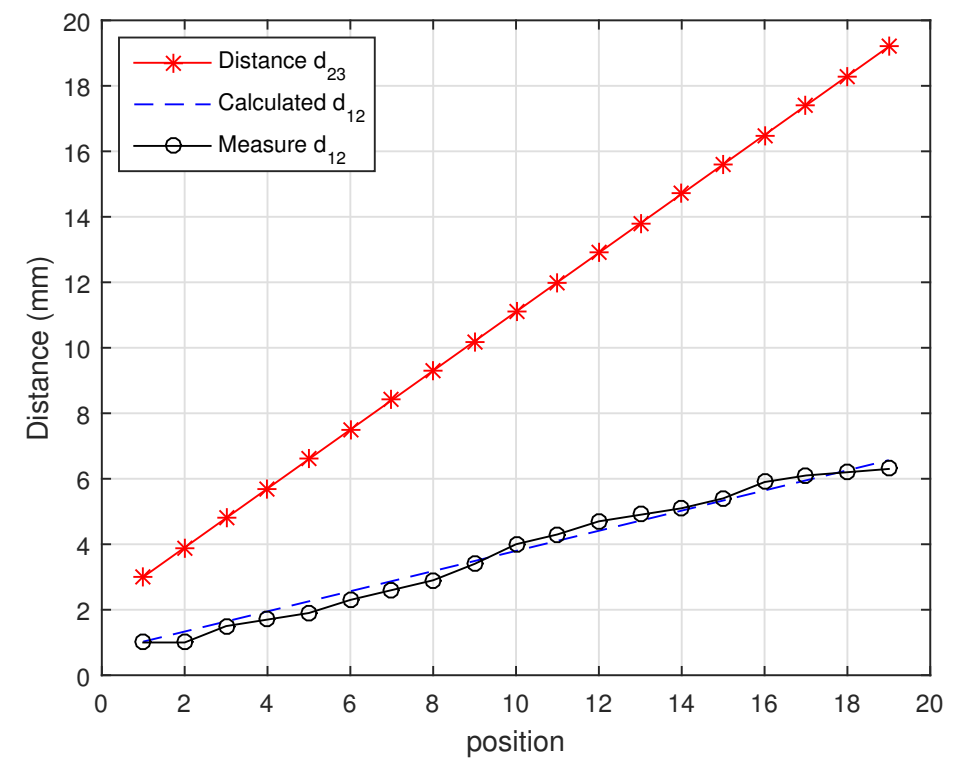

Figure 11. The relation between the distances $d_{12}$ and $d_{23}$ and a measured distance $d_{12}$.

The efficiency of the system was measured, combining the pre-measured and calculated data to achieve maximum efficiency. In Figure 12, the measured efficiency is compared to the calculated one of the system for multiple random distances, which fits well with the calculated pattern of $d_{12}$ adjustments. It is also evident that the system could demonstrate further higher efficiencies than the obtained maximum efficiency, at shorter distances than the higher distance. 


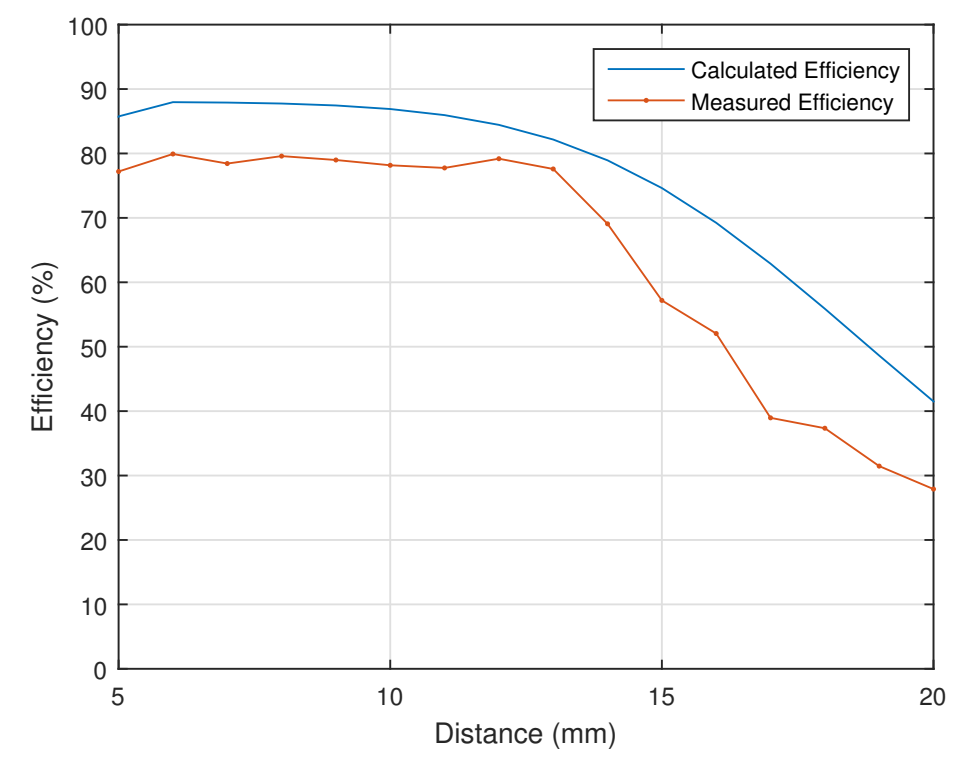

Figure 12. Comparison between the calculated and measured efficiencies of the system.

\subsection{Angle Coverage}

The position angle of the transmitter and receiver is also another crucial factor that may impact the system efficiency. In order to measure the efficiency, the angle of the receiver was constantly changed according to the angle of the transmitter, which varied between $0^{\circ}$ and $90^{\circ}$, as illustrated in Figure 13. The position of the receiver was also placed at various distances according to the transmitter. For instance, as shown in Figure 9, $\Delta d_{23}$ indicates the change of the distance between the RX' to the previous RX position, which is $1 \mathrm{~mm} . d_{23 \max }$ indicates the distance between the final RX position and the $\mathrm{Tx}$, which is $20 \mathrm{~mm}$ for our measurements.

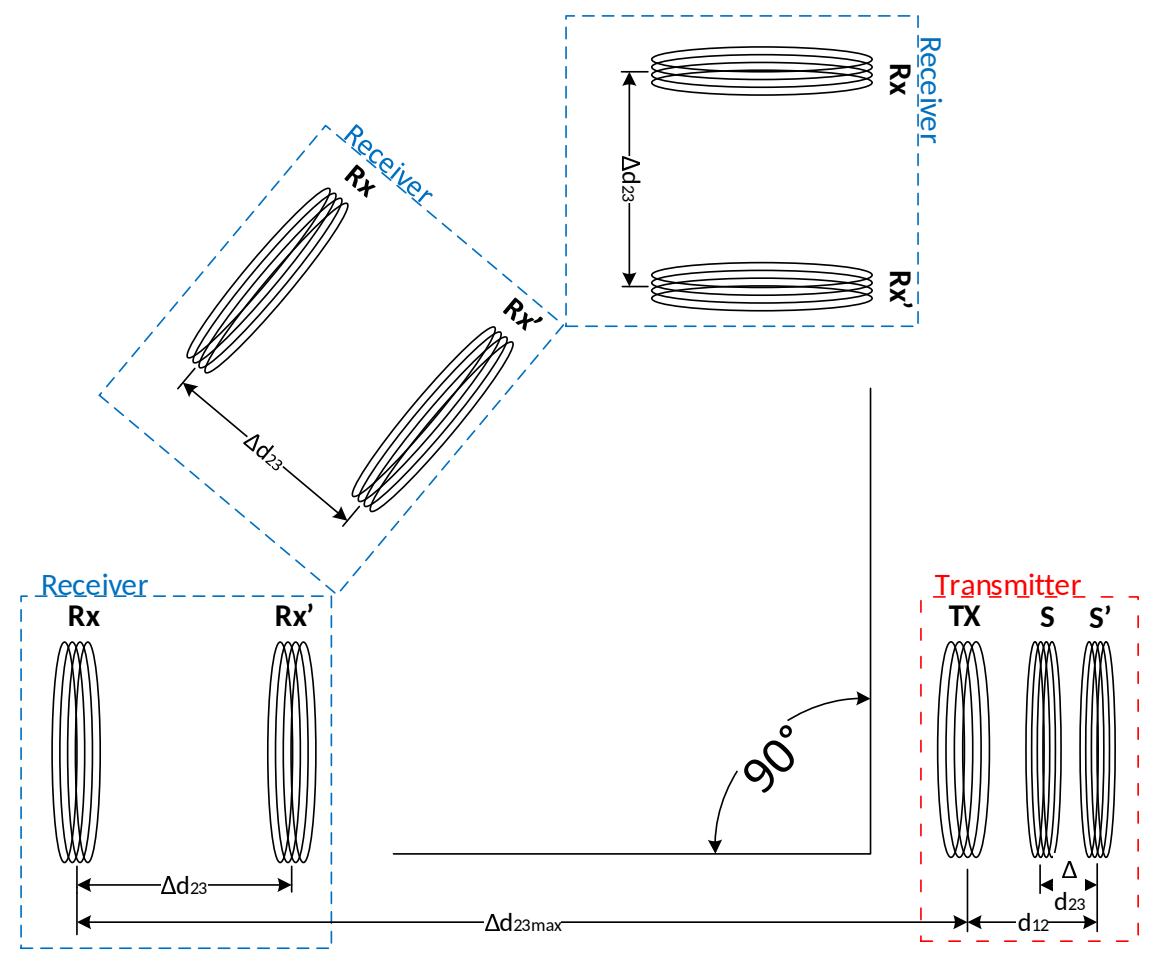

Figure 13. Positioning of receiver for the measurement of angle efficiency. 
Simultaneously, the distance between the Tx and $S$ was adjusted $\left(\Delta d_{12}\right)$ according to the changes made, distance-wise between the Tx and the RX $\left(\Delta d_{23}\right)$; see Figure 13. This further indicates the changes made to distance $d_{12}$ to achieve the highest efficiency according to the previous measurements. The receiver placement was changed for $30^{\circ}$ according to the previous measurements, and the measurement was repeated for new alignment.

As Figure 14 indicates, the maximum efficiency drops in accordance with the angle at which the transmitter is being placed. The greater the angle between transmitter and receiver, the lower the efficiency of the charger is and vice versa. It can also be seen that the angle above $30 \%$ highly affects the charging efficiency, as well as the distance at which the charger operates. Furthermore, the angle above $60 \%$ reduces the working range of the charger to a few millimetres and drastically decreases the efficiency.

From the measurements, it is evident that for the charger to have high efficiency and the highest possible distance covered, the angle placement must be lower that $30 \%$.

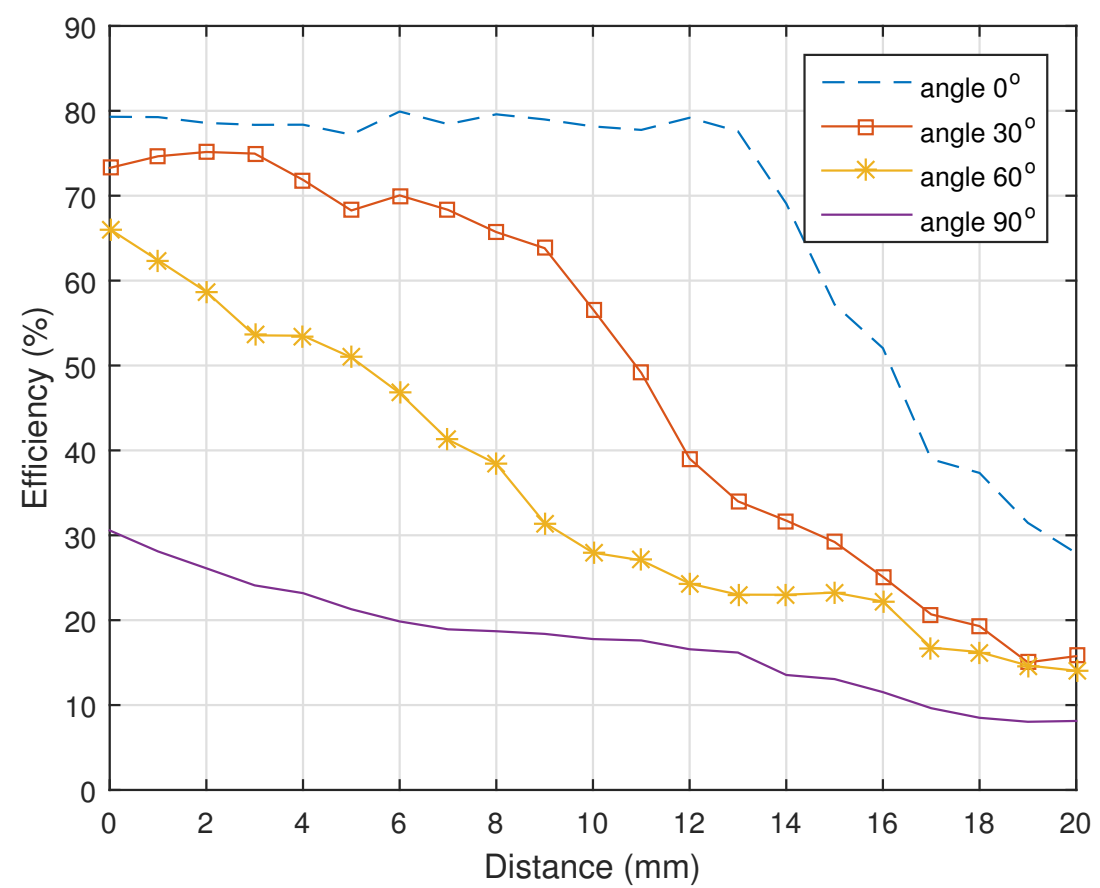

Figure 14. Measured angle efficiency of the combined system for various angles between transmitter and receiver.

\subsection{The Impact of the Coils' Misalignment}

A misalignment between the transmitter and receiver coils also plays an important role of relevance to the system efficiency. As presented in Figure 15, the measurements were carried out by changing the position of the coverage between the transmitter and receiver loops from $100 \%$ coverage down to $10 \%$. The measurements were commissioned at $0 \%, 50 \%, 70 \%$ and $90 \%$ misplacement. The distance between Tx and RX was also altered by $\Delta d_{23}=1 \mathrm{~mm}$, up to $d_{23 \max }$, which was equal to $20 \mathrm{~mm}$. The distance between Tx and $\mathrm{S}$ was also altered accordingly by $\Delta d_{12}$, in order to maximize the system efficiency.

As for the angle covered, the efficiency drops according to the misalignment that occurred. Interestingly, the effect on the efficiency due to the misalignment was indicated as up to $60 \%$, which does not seem to have massively impacted the overall system efficiency. However, over $60 \%$ misalignment within the coil structure has degraded the system efficiency drastically while misalignment over $90 \%$ has caused severe efficiency drops. 

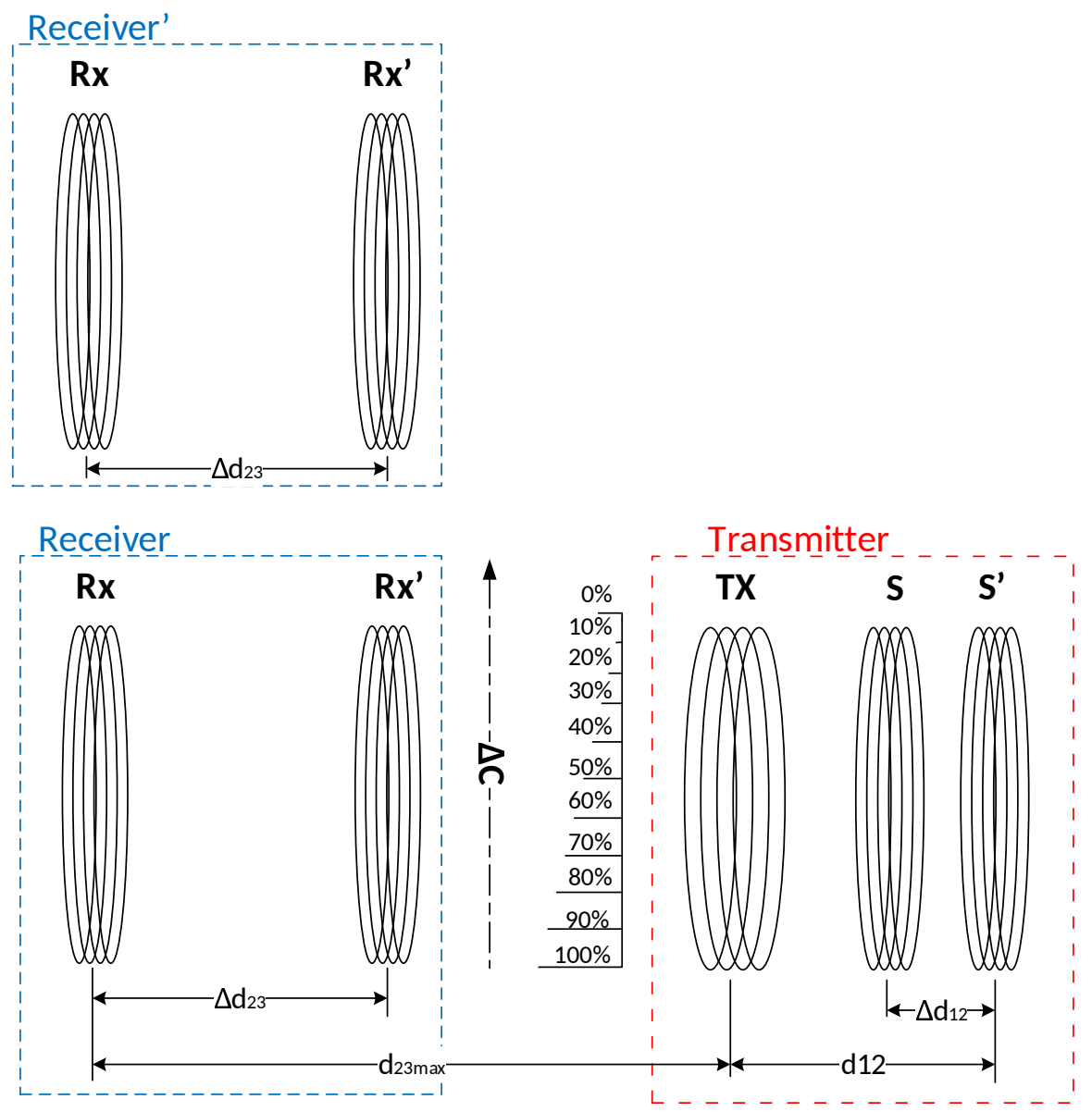

Figure 15. Positioning of the receiver for the measurement of the misalignment efficiency.

\section{Charger Efficiency Based on the Measured Results}

Our proposed wireless power charger design is based on real-time measurements. The charger efficiency is highly sensitive to both coil misalignment and the change of angle between the transmitter and receiver. Moreover, the charger efficiency possesses an inverse relationship with the misalignment and the change of angle. As measured, the maximum distance between the transmitter and receiver where the wireless charger demonstrates its high efficiency is $12 \mathrm{~mm}$ with an angle of $20 \%$. Based on the experimental results in Figure 16, a 50\% misalignment may cause approximately 15\% of an efficiency drop. From the previous calculation, it can be shown that the starting distance between TX and Rx will be $5 \mathrm{~mm}$.

The charger transmitter circuit adjusts the distance between the source and transmitter loops to fulfil the changing position requirements of the receiver. However, in order to perform the charging efficiently, the charger must be capable of adjusting the angle to reach the receiver, as shown in Figure 17.

Nevertheless, Figure 18 indicates that the proposed system exhibits a tolerance of misalignment up to a 50-mm radius within the charging pad where the efficiency remains at or above $60 \%$. According to calculations, the efficiency at a $50-\mathrm{mm}$ displacement will be $67.6 \%$, while the efficiency at full coverage is just above $79 \%$. Likewise, the overall efficiency loss remains in the range of $10 \%$, providing more flexibility, allowing the device to be charged efficiently within the $50-\mathrm{mm}$ radius. For the sake of comparison, Figure 18 also shows results for the conventional 2-coil system. It is clear that the proposed system always has better performance, and the improvement gap becomes more significant as the distance becomes larger. 


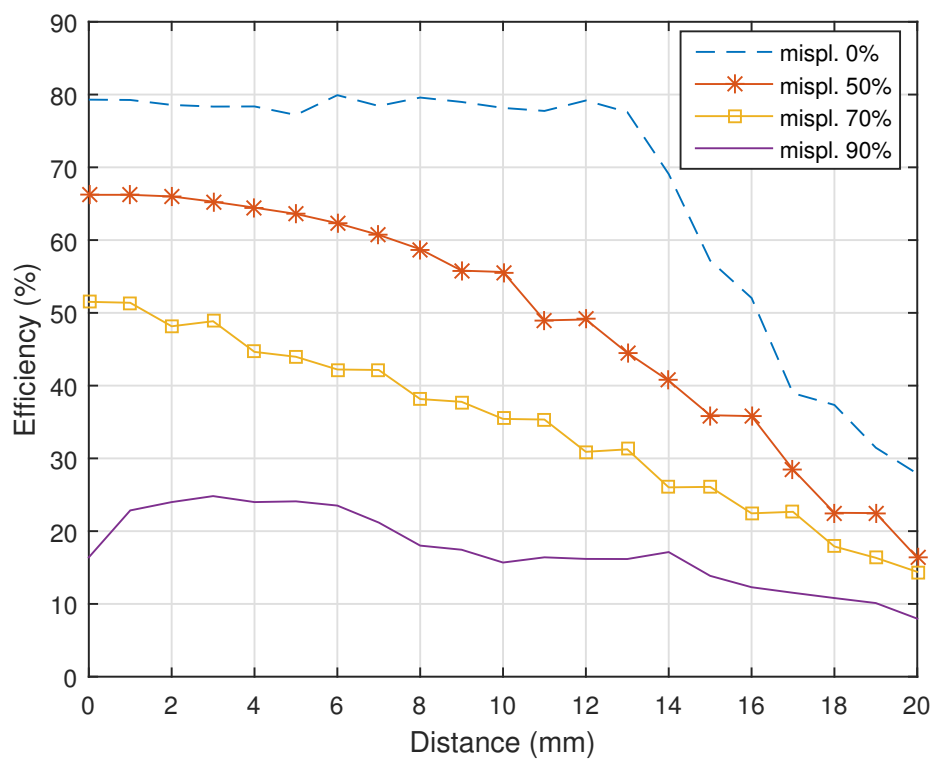

Figure 16. Measured misalignment efficiency of the combined system for various positions of the receiver.

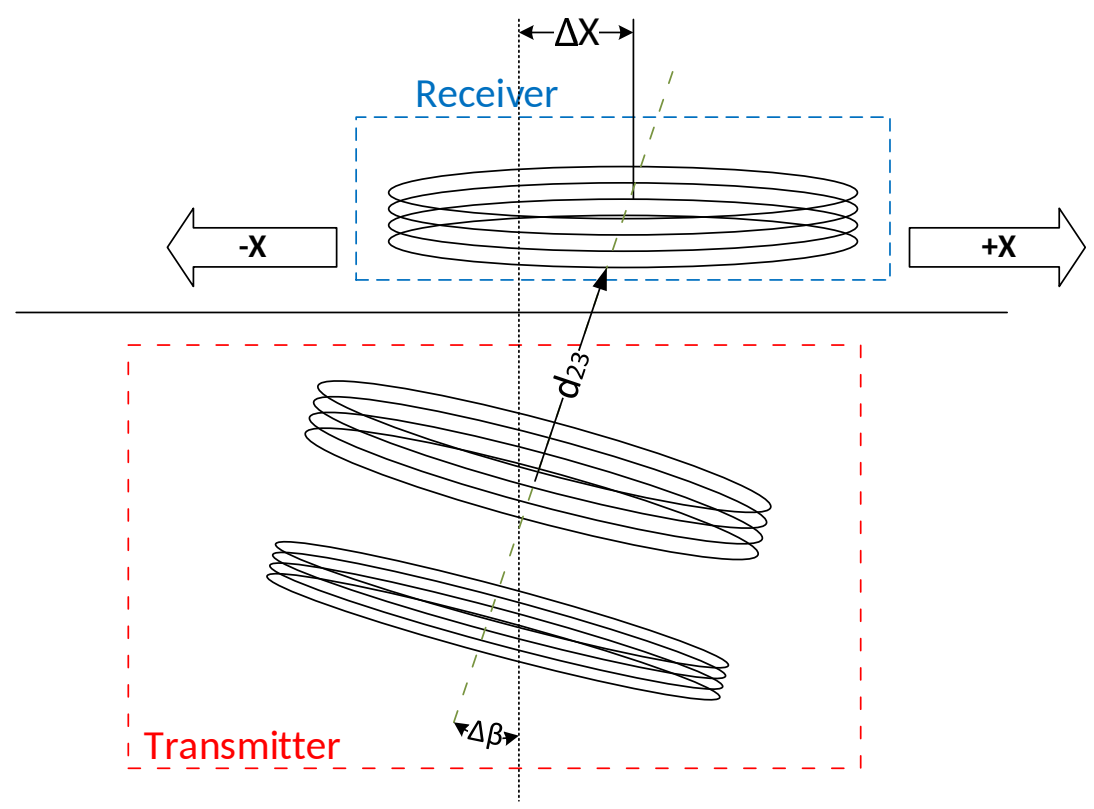

Figure 17. Diagram of the proposed WPT charger system.

At this end, it is worthwhile mentioning that the proposed system may not be always the best option in practical implementations due to its size. However, the improved efficiency and extended operating bandwidth of this system can marginalize the size issue. The other attractive advantage of the proposed configuration is its ability to perform well over shorter distances, which is a main drawback of other existing coil resonant systems. 


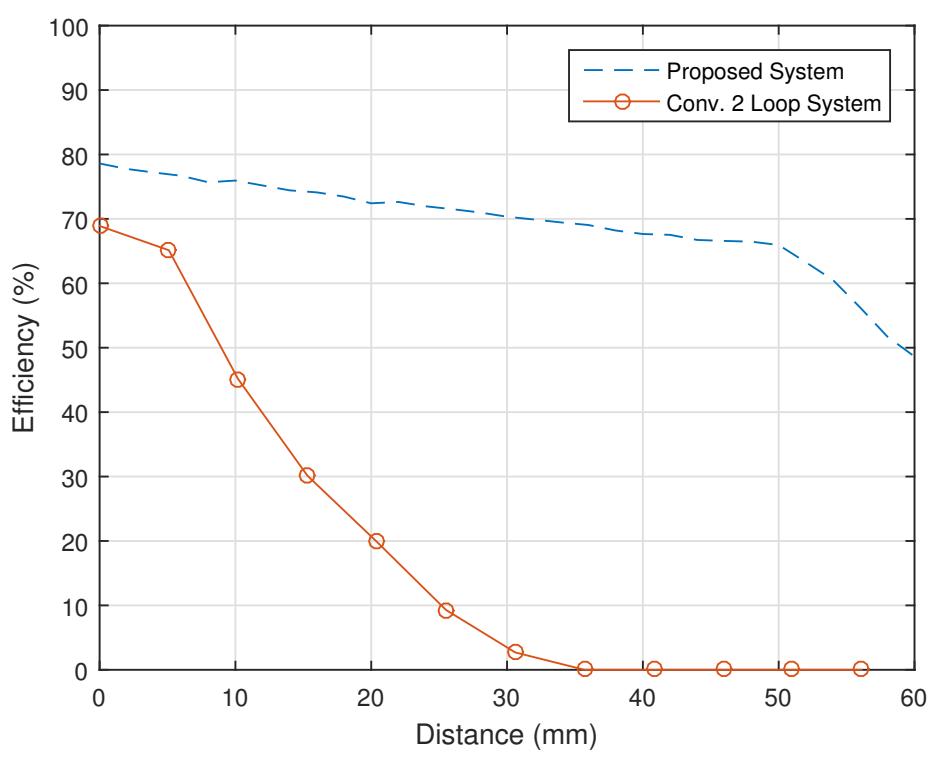

Figure 18. The efficiency of the proposed charger based on misalignment from the centre.

\section{Conclusions}

A hybrid SCMR-CSCMR system has been proposed in this paper. Varying the distance between the Tx and Rx loops can adversely impact the efficiency of WPT systems. However, the paper further explains that exploiting the variable distance between the source and Tx loops can be adjusted to meet the power transfer requirements, eliminating further performance penalties. Therefore, the proposed system adopted an adjustable SCMR concept at the transmitter side and the CSCMR model at the receiver end. This effort also illustrates that adaptively changing the distance between the source and Tx loops simultaneously aids the circuit to maintain the maximum efficiency throughout the whole transmission range provided, while the transmitting frequency remains unchanged. Further, the validated results also prove that the proposed charger provides better flexibility for devices to be charged within a 50-mm radius from the centre of the charging platform at a relatively high efficiency. Finally, the measured and calculated results tend to have similar results despite some differences, which resulted from the approximations used in our analysis.

Acknowledgments: This research has been carried out within the "Smart In-Building Micro Grid for Energy Management" project funded by EPSRC (EP/M506758/1) and supported by Innovate UK (Innovate UK Project 101836).

Author Contributions: Matjaz Rozman and Bamidele Adebisi conceived and designed the experiments. Matjaz Rozman performed the experiments. Matjaz Rozman and Michael Fernando analysed the data. Bamidele Adebisi and Khaled M. Rabie contributed materials and analysis tools. Matjaz Rozman and Khaled M. Rabie and Michael Fernando and Rupak Kharel wrote the paper. Augustine Ikpehai and Haris Gacanin reviewed the manuscript.

Conflicts of Interest: The authors declare no conflict of interest.

\section{References}

1. Rabie, K.M.; Adebisi, B.; Rozman, M. Outage probability analysis of WPT systems with multiple-antenna access point. In Proceedings of the 2016 10th International Symposium on Communication Systems, Networks and Digital Signal Processing (CSNDSP), Prague, Czech Republic, 20-22 July 2016; pp. 1-5.

2. Rabie, K.M.; Adebisi, B.; Alouini, M.S. Wireless power transfer in cooperative DF relaying networks with log-normal fading. In Proceedings of the 2016 IEEE Global Communications Conference (GLOBECOM), Washington, DC, USA, 4-8 December 2016; pp. 1-6. 
3. Moriwaki, Y.; Imura, T.; Hori, Y. Basic study on reduction of reflected power using DC/DC converters in wireless power transfer system via magnetic resonant coupling. In Proceedings of the IEEE International Telecommunications Energy Conference, Amsterdam, The Netherlands, 9-13 October 2011; pp. 1-5.

4. Li, Y.; Mai, R.; Lin, T.; Sun, H.; He, Z. A novel WPT system based on dual transmitters and dual receivers for high power applications: Analysis, design and implementation. Energies 2017, 10, 174.

5. Li, Y.; Wang, Y.; Cheng, Y.; Li, X.; Xing, G. QiLoc: A Qi wireless charging based system for robust user-initiated indoor location services. In Proceedings of the IEEE International Conference on Sensing, Communication, and Networking, Seattle, WA, USA, 22-25 June 2015; pp. 480-488.

6. Liu, X. Qi standard wireless power transfer technology development toward spatial freedom. IEEE Circuits Syst. Mag. 2015, 15, 32-39.

7. Van Wageningen, D.; Staring, T. The QI wireless power standard. In Proceedings of the International Power Electronics and Motion Control Conference, Ohrid, Macedonia, 6-8 September 2010; pp. 15-32.

8. Haldi, R.; Schenk, K.; Nam, I.; Santi, E. Finite-element-simulation-assisted optimized design of an asymmetrical high-power inductive coupler with a large air gap for EV charging. In Proceedings of the IEEE Energy Conversion Congress and Exposition, Denver, CO, USA, 15-19 September 2013; pp. 3635-3642.

9. Wen, F.; Huang, X. Optimal magnetic field shielding method by metallic sheets in wireless power transfer system. Energies 2016, 9, 733.

10. Haldi, R.; Schenk, K. A $3.5 \mathrm{~kW}$ wireless charger for electric vehicles with ultra high efficiency. In Proceedings of the IEEE Energy Conversion Congress and Exposition, Pittsburgh, PA, USA, 14-18 September 2014; pp. 668-674.

11. Chen, W.; Liu, C.; Lee, C.H.T.; Shan, Z. Cost-effectiveness comparison of coupler designs of wireless power transfer for electric vehicle dynamic charging. Energies 2016, 9, 906.

12. Kurs, A.; Karalis, A.; Moffatt, R.; Joannopoulos, J.D.; Fisher, P.; Soljačić, M. Wireless power transfer via strongly coupled magnetic resonances. Science 2007, 317, 83-86.

13. Daerhan, D.; Hu, H.; Georgakopoulos, S.V. Novel topologies of misalignment insensitive SCMR wireless power transfer systems. In Proceedings of the IEEE Antennas and Propagation Society International Symposium, Memphis, TN, USA, 6-11 July 2014; pp. 1341-1342.

14. Jolani, F.; Yu, Y.; Chen, Z. Enhanced planar wireless power transfer using strongly coupled magnetic resonance. Electron. Lett. 2015, 51, 173-175.

15. Wei, X.; Wang, Z.; Dai, H. A critical review of wireless power transfer via strongly coupled magnetic resonances. Energies 2014, 7, 4316-4341.

16. Liu, D.; Hu, H.; Georgakopoulos, S.V. Misalignment sensitivity of strongly coupled wireless power transfer systems. IEEE Trans. Power Electron. 2017, 32, 5509-5519,

17. Bao, K.; Hu, H.; Georgakopoulos, S.V. Design considerations of conformal SCMR system. In Proceedings of the IEEE Wireless Power Transfer Conference, Boulder, CO, USA, 13-15 May 2015; pp. 1-3.

18. Hu, H.; Bao, K.; Gibson, J.; Georgakopoulos, S.V. Printable and Conformal Strongly Coupled Magnetic Resonant systems for wireless powering. In Proceedings of the 2014 IEEE 15th Annual Wireless and Microwave Technology Conference (WAMICON), Tampa, FL, USA, 6 June 2014; pp. 1-4.

19. Gibson, J.; Bao, K.; Hu, H.; Georgakopoulos, S.V. Wireless charging for Li-Ion battery using a printable Conformal SCMR. In Proceedings of the IEEE Antennas and Propagation Society International Symposium, Toronto, ON, Canada, 11-17 July 2010; pp. 1349-1350.

20. Sample, A.P.; Meyer, D.T.; Smith, J.R. Analysis, experimental results, and range adaptation of magnetically coupled resonators for wireless power transfer. IEEE Trans. Ind. Electron. 2011, 58, 544-554.

21. Nair, V.V.; Choi, J.R. An efficiency enhancement technique for a wireless power transmission system based on a multiple coil switching technique. Energies 2016, 9, 156.

22. Beh, T.C.; Imura, T.; Kato, M.; Hori, Y. Basic study of improving efficiency of wireless power transfer via magnetic resonance coupling based on impedance matching. In Proceedings of the 2010 IEEE International Symposium on Industrial Electronics, Bari, Italy, 4-7 July 2010; pp. 2011-2016.

23. Hu, P.; Ren, J.; Li, W. Frequency-splitting-free synchronous tuning of close-coupling self-oscillating wireless power transfer. Energies 2016, 9, 491.

24. Park, J.; Tak, Y.; Kim, Y.; Kim, Y.; Nam, S. Investigation of adaptive matching methods for near-field wireless power transfer. IEEE Trans. Antennas Propag. 2011, 59, 1769-1773. 
25. Noriaki, O.; Kenichiro, O.; Hiroki, K.; Hiroki, S.; Shuichi, O.; Tasuku, M. Efficiency improvement of wireless power transfer via magnetic resonance using transmission coil array. In Proceedings of the 2011 IEEE International Symposium on Antennas and Propagation, Spokane, WA, USA, 3-8 July 2011; pp. 1707-1710.

26. Liu, F.; Yong, Y.; Jiang, D.; Ruan, X.; Chen, X. Modeling and optimization of magnetically coupled resonant wireless power transfer system with varying spatial scales. IEEE Trans. Power Electron. 2017, 32, 3240-3250.

27. Mou, X.; Groling, O.; Sun, H. Energy efficient and adaptive design for wireless power transfer in electric vehicles. IEEE Trans. Ind. Electron. 2017, doi:10.1109/TIE.2017.2686299.

28. Xie, X.; Bucknall, R.W.G.; Yearwood, K. Simulation study of a magnetic coupled resonant wireless energy transfer and storage system for electric vehicles under dynamic condition. In Proceedings of the Australasian Universities Power Engineering Conference, Brisbane, Australia, 25-28 September 2015; pp. 1-6.

29. Jonah, O.; Georgakopoulos, S.V. Wireless power transfer in concrete via strongly coupled magnetic resonance. IEEE Trans. Antennas Propag. 2013, 61, 1378-1384.

30. Kahng, S. Enhanced Coupling Structures for Wireless Power Transfer Using the Circuit Approach and the Effective Medium Constants (Metamaterials), Wireless Power Transfer Principles and Engineering Explorations. Available online: www.intechopen.com/books/wireless-power-transfer-principles-and-engineering-explorations / enhanced-coupling-structures-for-wireless-power-transfer-using-the-circuit-approach-and-the-effectiv (accessed on 6 January 2017).

31. Theilmann, P.T.; Asbeck, P.M. An analytical model for inductively coupled implantable biomedical devices with ferrite rods. IEEE Trans. Biomed. Circuits Syst. 2009, 3, 43-52.

32. Lu, X.; Wang, P.; Niyato, D.; Kim, D.I.; Han, Z. Wireless charging technologies: Fundamentals, standards, and network applications. IEEE Commun. Surv. Tutor. 2016, 18, 1413-1452.

33. Karalisa, A.; Joannopoulos, J.D.; Soljačić, M. Efficient wireless non-radiative mid-range energy transfer. Ann. Phys. 2008, 323, 34-48.

34. Bouattour, G.; Kallel, B.; Sasmal, K.; Kanoun, O.; Derbel, N. Comparative study of resonant circuit for power transmission via inductive link. In Proceedings of the IEEE International Multi-Conference on Systems, Signals \& Devices (SSD15), Sfax, Tunisia, 16-19 March 2015; pp. 1-6.

35. Hu, H.; Georgakopoulos, S.V. Design of optimal and broadband conformal SCMR systems. In Proceedings of the IEEE Antennas and Propagation Society International Symposium, Memphis, TN, USA, 6-11 July 2014; pp. 1345-1346.

36. Li, X.; Dai, X.; Li, Y.; Sun, Y.; Ye, Z.; Wang, Z. Coupling coefficient identification for maximum power transfer in WPT system via impedance matching. In Proceedings of the IEEE PELS Workshop on Emerging Technologies: Wireless Power Transfer, Knoxville, TN, USA, 4-6 October 2016; pp. 27-30.

37. Ramaswamy, V.; Edison, A.S.; Brey, W.W. Inductively-coupled frequency tuning and impedance matching in HTS-based NMR probes. IEEE Trans. Appl. Supercond. 2017, 27, 1502505.

38. Huang, Y.; Shinohara, N.; Mitani, T. Impedance matching in wireless power transfer. IEEE Trans. Microw. Theory Tech. 2016, 65, 582-590.

(C) 2017 by the authors. Licensee MDPI, Basel, Switzerland. This article is an open access article distributed under the terms and conditions of the Creative Commons Attribution (CC BY) license (http:/ / creativecommons.org/licenses/by/4.0/). 